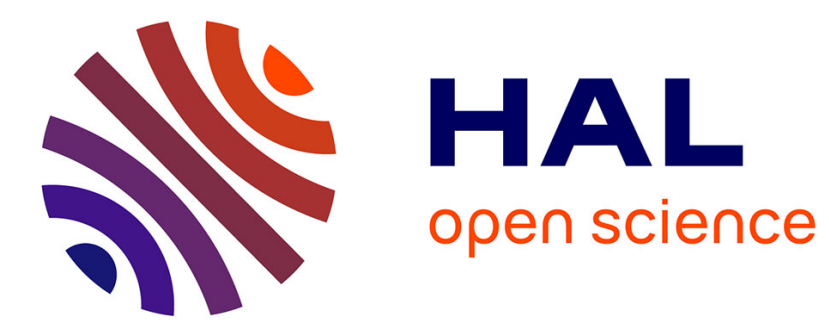

\title{
Rotational instabilities of the Earth induced by mantle density anomalies
}

\author{
Y. Ricard, R. Sabadini
}

\section{To cite this version:}

Y. Ricard, R. Sabadini. Rotational instabilities of the Earth induced by mantle density anomalies. Geophysical Research Letters, 1990, 17 (5), pp.627-630. 10.1029/GL017i005p00627 . hal-02046605

\section{HAL Id: hal-02046605 \\ https://hal.science/hal-02046605}

Submitted on 2 Feb 2021

HAL is a multi-disciplinary open access archive for the deposit and dissemination of scientific research documents, whether they are published or not. The documents may come from teaching and research institutions in France or abroad, or from public or private research centers.
L'archive ouverte pluridisciplinaire HAL, est destinée au dépôt et à la diffusion de documents scientifiques de niveau recherche, publiés ou non, émanant des établissements d'enseignement et de recherche français ou étrangers, des laboratoires publics ou privés. 


\title{
ROTATIONAL INSTABILITIES OF THE EARTH \\ INDUCED BY MANTLE DENSITY ANOMALIES
}

\author{
Yanick Ricard \\ Département de Géologie, Ecole Normale Supérieure, Paris, France \\ Roberto Sabadini \\ Dipartimento di Fisica, Settore Geofisica, Università di Bologna, Italy
}

\begin{abstract}
From the seismic tomographic models, we estimate the offset between the geographic pole and the main inertial axis. This offset, which is large for a static Earth, is drastically reduced when the dynamical behaviour of the mantle is taken into account. For a mantle stratifcation with a viscosity increase by a factor of about 50 , this offset is comparable to what can be estimated on the basis of remanent post-glacial depression. We also discuss the rate of polar drift induced by both internal mass heterogeneities and Pleistocenic deglaciation. Contributions from internal sources and ice-sheets disintegration are consistent with the observed secular trend. For high viscosity contrasts, our results are quite insensitive to the phase transition or chemical nature of the upperlower mantle interface. This study shows that there is no contradiction between the tomographic models of the Earth and their implications for the rotational stability of our planet; our findings also agree with recent analyses of paleomagnetic data.
\end{abstract}

\section{Introduction}

Seismic tomography reveals the distribution of density anomalies within the upper and lower mantle [Driewonski, 1984; Woodhouse and Driewonski, 1984]. When the inertia tensor associated to this density distribution is evaluated, an offset remains between the geographic pole and the principal axis of inertia. This offset is orders of magnitude larger than the observed excursions of the rotation pole. Tanimoto [1989] suggested that this is not reasonable. However, Chao emphasizes in his comment [1989] that such a comparison between this geometrical offset and the excursion of the rotation axis, which is of dynamical concern, is inappropriate.

In this paper we address again this problem showing that there is no contradiction between the seismic tomographic results and rotational data if we account for 1)the effects of dynamic compensation of density anomalies and 2)-the rotational behaviour of the planet. Our work is somewhat akin to the discussion by Munk and MacDonald [1960] on the possible polar drift induced by the oceancontinent distribution. A comparison is also drawn with post-glacial rebound to emphasize the similarities and differences in the geophysical processes associated with surface and internal mass redistributions.

\section{Inertia and tidal deformation of a dynamic Earth}

The computation of the Earth's inertial tensor must take into account the existence of internal density structure. In addition, these mass heterogeneities drive a Copyright 1990 by the American Geophysical Union.

Paper number 90GL00630

$0094-8276 / 90 / 90$ GL-00630\$03.00 mantle flow which deflects the various interfaces such as the surface, the core-mantle boundary or the upperlower mantle interface. The induced surface topography is small and cannot been distinguished from an isostatically compensated topography at the Moho. However, the mass associated with this topography is comparable to the one of the deep forcing heterogeneity and its contribution to the inertia tensor cannot be neglected.

Let us assume that the internal mass heterogeneities are described by a spherical harmonic expansion of the form

$$
\rho(r, \theta, \phi)=\sum_{l m} \rho_{l m i}(r) Y_{l}^{m i}(\theta, \phi)
$$

where $\theta$ is the colatitude and $\phi$ the longitude. The superscript $i$ distinguishes between the cosine $(i=c)$ and sine $(i=s)$ part of the spherical harmonics. The mass deflections induced at the $j$ chemical interfaces can be represented by surface mass distributions $\delta \sigma\left(r_{j}, \theta, \phi\right)$. Previous works have shown that these topographies can be deduced using a Green function formalism [e.g. Ricard et al., 1984; Richards and Hager, 1984]. We define $\delta \sigma_{l m i}\left(r_{j}\right)$ as the component of degree $l$ and order $m$ of the surface mass heterogeneity induced at the depth $j$. We have

$$
\delta \sigma_{l m i}\left(r_{j}\right)=\int_{0}^{a} T_{l, j}(r) \rho_{l m i}(r) d r
$$

where $a$ is the Earth radius and $T_{l, j}(r)$ the Green function for the topography with degree $l$ induced at the interface $j$. The problem of the rotation of the Earth involves the inertia terms which are only related to the degree 2. The relationships between these quantities and the mass distribution read

$$
I_{\alpha \beta}=\int_{0}^{a} G(r) \rho_{\alpha \beta}(r) d r
$$

where $a$ is the Earth radius, $\alpha$ and $\beta$ stand for $x, y$ or $z$, $\rho_{\alpha \beta}$ are linear functions of the $\rho_{2 m i}$ [Tanimoto, 1989] and $G(r)$ is the inertia Green function defined by:

$$
G(r)=\frac{1}{a^{4}}\left(r^{4}+\sum_{j} T_{2, j}(r) r_{j}^{4}\right)
$$

The last terms neglected by Tanimoto [1989] take into account the dynamic behaviour of the viscous Earth; they drastically change the behaviour of our planet. When a mass anomaly reaches a chemical interface $j$, the induced surface topography $T_{l, j}$ tends to -1 , whereas the other deflections vanish. The Green functions are thus zero at each interface. They are depicted in Figure 1 for lower mantle viscosities $1,10,50$ or 100 times the upper mantle one: A $100 \mathrm{~km}$ thick lithosphere 100 times more viscous than the upper mantle is also present. Free-slip boundary conditions have been applied at the surface and at the core-mantle boundary. Figure $1 \mathrm{a}$ is for the case 


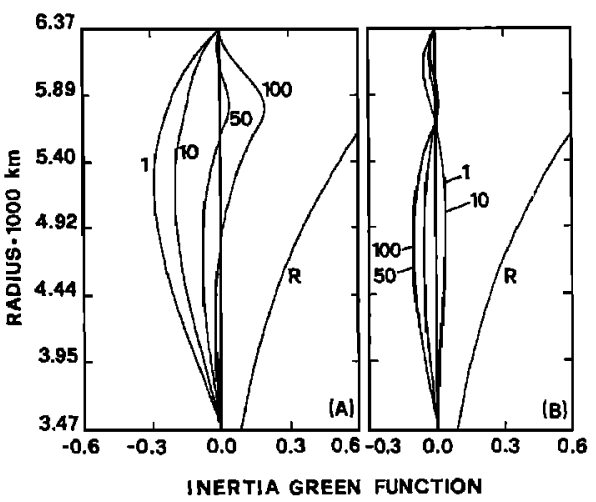

Fig. 1. Inertia Green functions. In (a), a phase transition occurs at $650 \mathrm{~km}$ depth, whereas in (b), the two parts of the mantle are chemically distinct. On each panel, the four curves correspond to a viscosity increase at $650 \mathrm{~km}$ depth by a factor of $1,10,50$ or 100 . The curves labelled $R$ are for a rigid Earth.

of a phase-transition at $650 \mathrm{~km}$ depth whereas in Figure $1 \mathrm{~b}$ a new interface at this depth allows for a possible chemical transition. With respect to the rigid Earth (R), the compensation of mass anomalies drastically reduces the amplitude of the inertia Green function. Viscosity increase in the lower mantle can produce a change in sign in the response. For large viscosity ratios, similar magnitudes are predicted for both chemical and phasetransition models.

The existence of non-diagonal inertia products leads to polar drift, which is controlled by the ability of the planet to readjust its equatorial bulge by viscous yielding. Polar drift generates variations of the centrifugal potential that induce, by rotational deformation, an extra contribution to the moments of inertia. These new offdiagonal components of the inertia tensor can be properly quantified in terms of tidal boundary conditions applied at the surface. The adimensional radial and tangential displacements describing the deformation of the planet induced by centrifugal forces are shown in Figure 2. We consider the same mantle stratifications as in Figure 1. A large viscosity increase at $650 \mathrm{~km}$ favours a channel effect in the upper mantle. This is enhanced by the presence of a chemical boundary (c and d).

\section{Tomographic models and rotation of the Earth}

We evaluate the inertia tensor components associated with the 3-dimensional structure inverted from seismic tomography models M84C [Woodhouse and Driewonski, 1984] and L02.56 [Driewonski, 1984]. We choose the following relationships between density and velocity anomalies $d \ln \rho / d \ln V_{s}=0.4$ and $d \ln \rho / d \ln V_{p}=0.5$.

In Table 1, we provide for our different models, the colatitude $\theta$ and longitude $\phi$ in the usual geographic coordinates of the maximum moment of inertia due to the internal sources only (central columns). In the right columns we account for the presence of the hydrostatic equatorial bulge. It must be emphasized that these inertia perturbations do not include the contributions due to variations in the centrifugal potential. The Earth is presently

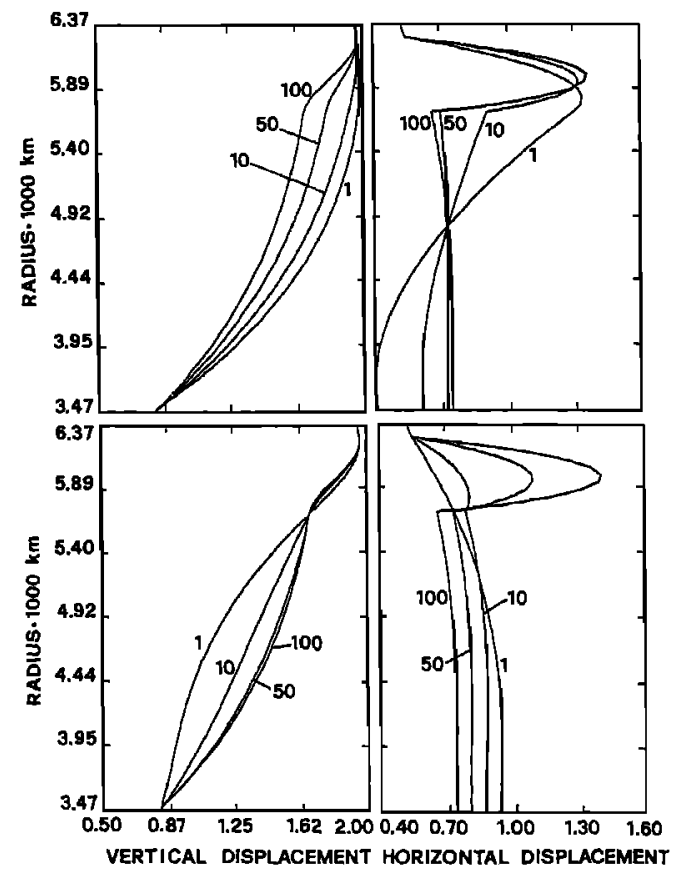

Fig. 2. The four panels depict the horizontal (right col umn) and vertical (left column) normalized displacements induced by a tidal load of degree 2 applied at the surface. Top panels correspond to a phase change at $650 \mathrm{~km}$ depth bottom panel stand for a chemical interface. The four viscosity jumps are those of Figure 1.

wandering toward the direction given in the right columns, but ultimately should reach the equilibrium configuration corresponding to the central columns. Except for the rigid Earth or for the phase transition model with a very high viscosity contrast, $\left(\nu_{2} / \nu_{1}=100\right)$, we find that seismic tomography gives a striking agreement between the maximum moment of inertia and the rotation pole. This could have been guessed from the good correlation between the observed $l=2$ geoid which has an equatorial maximum, and the geoid deduced from lower mantle tomography. Furthermore, the good agreement between the computed and observed $C_{22}$ and $S_{22}$ components of the geoid related to the least inertia axis has been already addressed [e.g. Hager et al., 1985; Ricard et al., 1989].

Table 1. Orientation in degrees of the principal inertia axis deduced from seismic tomography using different mantle stratifications.

\begin{tabular}{crrrrr}
\hline & \multicolumn{2}{c}{ masses } & \multicolumn{2}{c}{ masses+bulge } \\
& visc. & colat. & long. & colat. & long. \\
\hline & 1 & 2.7 & 163.5 & .19 & 171.2 \\
phase & 10 & 2.4 & 161.6 & .13 & 168.5 \\
change & 50 & .9 & 63.8 & .01 & 61.7 \\
& 100 & 85.8 & 51.6 & .12 & 2.5 \\
& 1 & 1.3 & 106.5 & .02 & 100.8 \\
chemical & 10 & 1.0 & 127.4 & .02 & 129.4 \\
interface & 50 & .9 & 137.6 & .02 & 141.8 \\
& 100 & .9 & 139.4 & .02 & 143.8 \\
rigid Earth & & 83.9 & 52.1 & .67 & 21.5 \\
\hline
\end{tabular}


In Figure 3 the radial logarithmic scale provides the values of the quantity $\left(I_{x x}^{2}+I_{y z}^{2}\right)^{1 / 2}$. The aximuth of the axis of maximum moment of inertia associated with the internal sources and equatorial bulge, is shown by the black dots. The contours give the error in the estimate of the inertia products due to an assumed depth independent uncertainty in the seismic velocity model of $20 \%$ of the maximum velocity anomaly. Because of the various changes of sign in both the inertia Green functions and the radial density variations, the resulting errors are fairly large. The R-dot corresponds to the rigid Earth [Taninoto, 1989], while our results are given in terms of the viscosity contrast $\nu_{2} / \nu_{1}$. For both phase-change and chemical transition models (panel $a$ and $b$ respectively), dynamic compensation produces a reduction in the inertia perturbation which can be as high as 50 . The azimuth is very sensitive to the rheological stratification. For the phasetransition, we observe a clockwise rotation of around $180^{\circ}$ for the high viscosity model $\left(\nu_{2} / \nu_{1}=100\right)$ with respect to the cluster corresponding to smoother viscosity contrasts. In the same reference system, we show for comparison the present values of the inertia perturbations induced by disintegration of Laurentide and Fennoscandia ice-sheets, which is assumed to have occurred 12,000 $\mathrm{yr}$ ago (white dots). For the glacial events we make use of a viscoelastic model [Sabadini et al., 1984]. We emphasize that, for the chemical boundary or high viscosity contrast in the phasetransition model, the inertia perturbations due to internal masses are comparable in magnitude with those predicted by another well documented geophysical process, such as the disintegration of large ice-sheets. This also shows that Tanimoto's argument [1989] of too large an offset between the axis of rotation and the axis of inertia of the Earth can hardly be sustained.

We now match the perturbations in the inertia tensor with the appropriate linearized Liouville equations, in
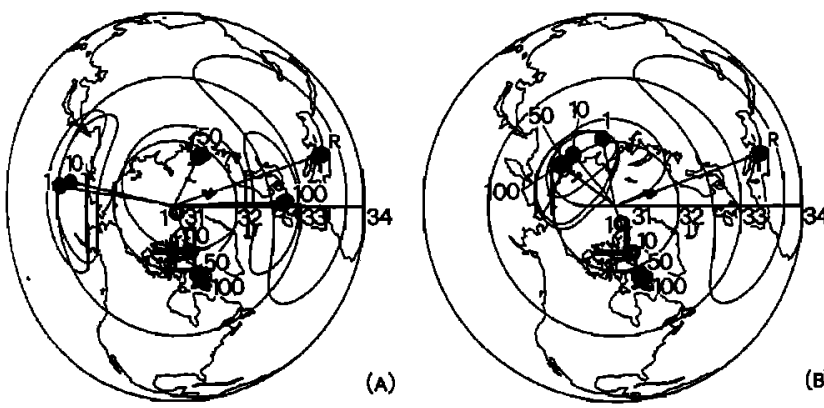

Fig. 3. Non diagonal inertia terms $I_{x z}$ et $I_{y z}$ due to internal loads (black dots) and Pleistocenic deglaciation (white dots). In (a) the Earth's mantle presents a phase transition at $650 \mathrm{~km}$ depth, in (b) a chemical interface. The viscosity jumps are given on the figure. A rigid Earth would have lead to the offset labelled $R$. The azimuth of the computed inertia axis is indicated by the geographic map and the amplitudes can be read radially on a logarithmic scale. The center corresponds to an amplitude of $10^{31} \mathrm{~kg} \cdot \mathrm{m}^{2}$ and the external circle to $10^{34} \mathrm{~kg} \cdot \mathrm{m}^{2}$. The estimated uncertainties are also indicated. order to draw a comparison between the predictions of our Earth models and the observed secular components of the polar motion. The steady state linearized version of Liouville equations for a stratified viscoelastic Earth take the following form [Sabadini et al., 1984]

$$
\left(\dot{m}_{1}, \dot{m}_{2}\right)=\frac{\mathbf{A}_{1}}{(C-A)}\left(I_{x z}, I_{y z}\right)
$$

where $\dot{m}_{1}, \dot{m}_{2}$ denotes the time derivatives of the director cosines of the axis of rotation and $C-A$ stands for the equatorial bulge. The residue $\mathbf{A}_{1}$ generalizes, for a stratified, viscoelastic Earth, the secular term used by Munk and Macdonald [1960] to estimate the polar drift driven by the distribution of the continents. Of course, a full treatment of this dynamical process would require to account for the trade-off between the time-dependence of the anomalous density structure and the relaxation times of both the isostatic modes (time-dependent deformations of the Earth due to internal masses) and rotational modes (time dependent deformations of the Earth due to changes in the centrifugal potential). This is beyond the scope of the present letter. $A_{1}$ is a very sensitive function of the mantle stratification [Sabadini and Yuen, 1989]. This is shown in Figure 4, where $A_{1}$ is given as a function of

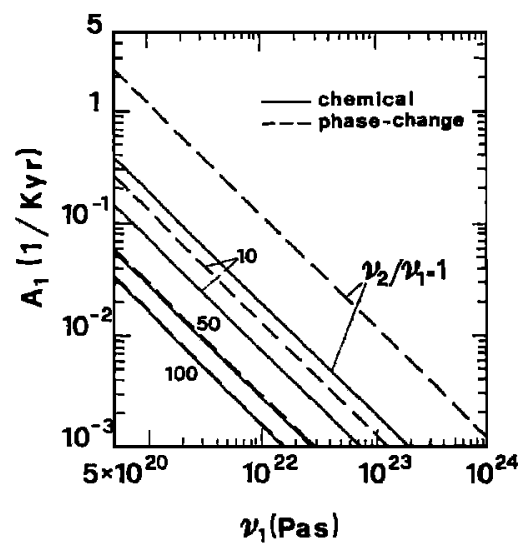

Fig. 4. Amplitude of the $\mathbf{A}_{1}$ factor as a function of the upper mantle viscosity for our 4 different viscosity jumps at $650 \mathrm{~km}$ depth. This interface can be a phase transition (dashed) or a chemical boundary (solid).

upper mantle viscosity $\nu_{1}$ for different ratios $\nu_{2} / \nu_{1}$. For viscosity contrasts of at most one order of magnitude, phase-change models (dashed), predict faster polar drift than corresponding chemically stratified models (solid). For larger viscosity contrasts, $\mathbf{A}_{1}$ does not depend on the nature of the boundary conditions at the upper-lower mantle interface. In Figure 5 we show in units of degrees per million year the rates of polar drift $\dot{m}$ with their uncertainties. They are derived from equation (5) for different mantle stratifications; panels $a$ and $b$ correspond to phase-change and chemical transition respectively. The star stands for the secular trend observed in the International Latitude Service (I.L.S.) data [Dickman, 1977]. In agreement with previous results [Nakada and Lambeck, 1989], we choose $\nu_{1}=5 \times 10^{20} \mathrm{~Pa} \cdot s$ in the upper 

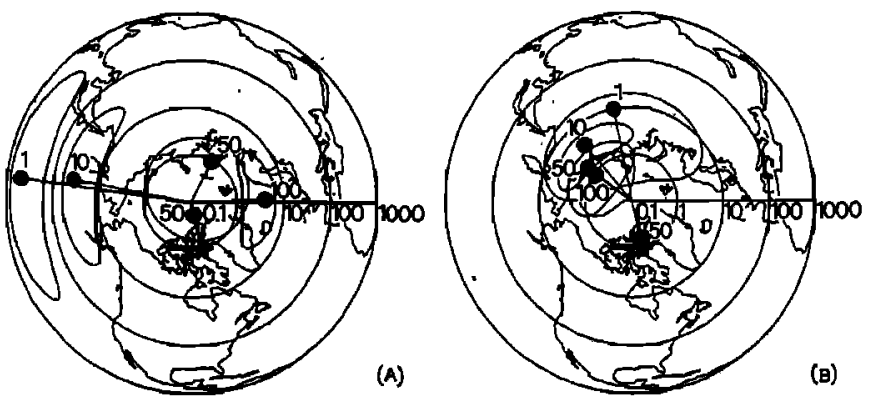

Fig. 5. Velocities of the polar drift induced by internal masses (black dots) or post glacial rebound (white dot) with the same representation as in Figure 3. The observed drift from I.L.S data is depicted by a star. The amplitudes can be read radially on a logarithmic scale from $.1 \mathrm{deg} / \mathrm{Myr}$ to $100 \mathrm{deg} / \mathrm{Myr}$.

mantle. The isoviscous, phase-change model predicts a rate of polar drift which is two orders of magnitude larger than the observed one; this quantity is generally lower for chemically stratified models. For $\nu_{2} / \nu_{1}=50$, the contribution of the glacial forcing (open circle) has been computed accounting for the complete set of isostatic and rotational modes [Sabadini et al., 1984]. Within the error bars, the contributions due to internal masses and glacial forcing, are not in contradiction with the observed I.L.S. data. The amplitude of the polar drift velocity, of about $1 \mathrm{deg} / \mathrm{Myr}$ or less is also consistent with recent reanalyses of paleomagnetic data, which point to the possibility of significant shift of the rotation pole in the late Cretaceous [Gordon and Livermore, 1987].

\section{Concluding remarks}

Our study indicates that the azimuth of the axis of maximum moment of inertia due to internal mass heterogeneities and its deviation from the rotation pole are very sensitive to the mechanical stratification of the mantle. In particular, the offset between the axes of rotation and maximum moment of inertia, is drastically reduced for realistic dynamical models of the planet. We stress the fact that the main inertial axis deduced from seismic tomography models, even without the contribution of the equatorial bulge, is generally very close to the Earth pole of rotation. When the off-diagonal components of the inertia tensor associated with the dynamically compensated mass anomalies are coupled with the long-term polar motion equations, we find that, within their uncertainties, the rates of polar drift induced by internal sources are not in contradiction with the observed secular trend in the I.L.S. data. In general for low viscosity increase, chemically stratified models must be preferred, but a phasechange boundary at the 650 seismic discontinuity also agrees with rotation data, provided a viscosity increase by a factor of about 50, is allowed in the modelling; this finding is consistent with recent estimates by Nakada and Lambeck [1989] derived from late Pleistocene and Holocene sea-level variations. For such a large viscosity contrast, phase-change and chemical transitions are essentially indistinguishable.
Acknowledgements. One of us (Y. Ricard), is supported by an E.S.A. fellowship. This work has also been partially supported by A.S.I. grant $N^{0} 88020$.

\section{References}

Chao, B. F., Comment on "Moment of inertia of threedimensional models of the Earth" by Toshiro Tanimoto, Geophys. Res. Let., 16, 1075, 1989.

Dickman, S. R., Secular trend on the Earth's rotation pole: consideration of motion of the latitude observations, Geophys. J. R. Astron. Soc., 51, 229-244, 1977.

Dziewonski, A. M., Mapping of the lower mantle: determination of lateral heterogeneity in $P$ velocity up to degree and order 6, J. Geophys. Res., 89, 5929-5952, 1984.

Gordon, R. G. and R. A. Livermore, Apparent polar wander of the mean lithosphere reference frame, Geophys. $J$. R. astr. Soc., 91, 1049-1057, 1987.

Hager, B.H., R. W. Clayton, M. A. Richards, R. P. Comer, and A. M. Dziewonski, Lower mantle heterogeneity, dynamic topography and the geoid, Nature, $319,541-$ $545,1985$.

Munk, W.H. and G. J. F. MacDonald, The rotation of the Earth, Cambridge University Press, New York, 1960.

Nakada, M. and K. Lambeck, Late Pleistocene and Holocene sea-level change in the Australian region and mantle rheology, J. Geophys. Res., 96, 497-517, 1989.

Ricard, Y., L. Fleitout, and C. Froidevaux, Geoid heights and lithospheric stresses for a dynamic Earth, Annales Geophysicae, 2, 267-286, 1984.

Ricard, Y., C. Vigny, and C. Froidevaux, Mantle heterogeneities, geoid, and plate motion: a Monte Carlo inversion, J. Geophys. Res., 94, 13739-13754, 1989.

Richards, M. A., and B. H. Hager, Geoid anomaly in a dynamic earth, J. Geophys. Res., 89, 5987-6002, 1984.

Sabadini, R., D. A. Yuen, and E. Boschi, A comparison of the complete and truncated versions of the polar wander equations, J. Geophys. Res., 89, 7609-7620, 1984.

Sabadini, R., and D. A. Yuen, Mantle stratification and long-term polar wander, Nature, s99, 373-375, 1989.

Tanimoto, T., Moment of inertia of three-dimensional models of the Earth, Geophys. Res. Let., 16, 389-392, 1989.

Woodhouse, J. H., and A. M. Dziewonski, Mapping the upper mantle: three-dimensional modeling of Earth structure by inversion of seismic waveforms, J. Geo. phys. Res., 89, 5953-5986, 1984.

Y. Ricard, Département de Géologie, Ecole Normale Supérieure, 24, rue Lhomond, 75231 Paris Cedex 05. R. Sabadini, Dipart. di fisica, Sett. di Geofisica, Univ. di Bologna, Viale Berti Pichat 8, 40127 Bologna, Italy.

(Received December 18, 1989; revised February 8, 1990; accepted February 16, 1990) 